\title{
Chemical Binding of Chitosan and Chitosan Nanoparticles onto Oxidized Cellulose
}

\author{
Olivera Sauperl, Mirjana Kostic, Jovana Milanovic, Lidija Fras Zemljic \\ Faculty of Mechanical Engineering, Maribor, Slovenia SLOVENIA \\ Correspondence to: \\ Olivera Sauperl email: olivera.sauperl@um.si
}

\begin{abstract}
The aim of this study was to analyze binding of chitosan and chitosan nanoparticles onto cellulose via oxidized cellulose.

The ability of chitosan and chitosan nanoparticles to be adsorbed onto surfaces was determined by the use of the XPS spectroscopy which provided information about chemical composition of the fiber surface. On the other hand, the gravimetric method was also used by which the amount of chitosan and chitosan nanoparticles bounded onto surface was calculated based on the difference in masses before and after functionalization. The most important was to study the influence of aldehyde groups on the stability of chitosan binding onto cellulose. Thus, desorption of chitosan/chitosan nanoparticles from the fiber surfaces was evaluated by the presence of total nitrogen (TN) in desorption bath as well as by polyelectrolyte titrations. Together with these two methods, desorption was evaluated also by gravimetric method, where the extent of desorption was evaluated on the basis of the differences in the masses of fibers before and after desorption.
\end{abstract}

It is concluded that the chitosan and chitosan nanoparticles are more efficiently bounded onto oxidized cellulose in comparison with the nonoxidized (reference) ones. Despite the binding of the positively-charged amino groups with the negative groups of cellulose and consequently smaller amount of available/residual protonated amino groups that are responsible for bioactivity, such functionalized fibers are still specifically antimicrobial.

Keywords: cellulose, oxidized cellulose, oxidation, chitosan, chitosan nanoparticles, FTIR, XPS, antimicrobial functionalization

\section{INTRODUCTION}

The demand for high-quality hygienic and medical textile products is increasing due the higher standard of living. Different methods of natural and regenerated cellulose material functionalization exist in order to achieve biologically-active effects. These are the oxidation processes, synthesis of antimicrobial cellulose, inclusion of metal nano particles into fibers such as silver, zinc, and copper, antimicrobial coating such as chitosan, benzophenone and addition of antimicrobial reagent into cellulose xanthate such as triclosan [1]. Most of these methods/reagents are controversial for humans and the environment due to inorganic salts, phenols and thiophenoles, antibiotics, and formaldehyde derivatives, which are used during production [2-3]. Therefore, the increasing tendency of research is seen where the functionalization is performed by the use of non-toxic, biodegradable, and environmentallyfriendly reagents [4-6].

These procedures include alternative and so far less used polysaccharides and their derivatives such as chitosan, which is a very interesting polysaccharide in the sense of fiber-forming polymer functionalization. It is a poly-cationic, renewable, and biodegradable polymer known for its wide-range of biological activities, including anti-microbial and antimycotic [7]. It also displays good bio-adhesive properties [8-12].

The trend in the use of chitosan is oriented towards the direction of chitosan nanoparticle use due to relatively high-specific surface area resulting in high biological efficiencies of these compounds. In the case of chitosan and/or chitosan nanoparticles presented onto the surface of the fibers, irreversible binding is in the case of conventional application of (washable) textiles more appreciated due to more permanent efficiency (long-term action) at the fiber interface. This is only possible in the case when suitable functional groups are presented within the fiber structures as binding places for the amino groups of chitosan or chitosan nanoparticles. For example, it is extremely important for irreversible attachment to introduce carboxyl or aldehyde groups into/onto the fibers which are then potential binding places for efficient chitosan adsorption. In this way, 
with carboxylic groups electrostatic attraction is assured between cellulose fibers as host material and chitosan as adsorbent. The most appropriate treatments of cellulose fibers for permanent chitosan binding are those which introduce aldehyde groups. In this way chitosan is chemically attached onto cellulose, which was proved by Strnad et al [13].

The aim of this research was focused towards the chemical modification of cellulose, more precisely to the oxidation process with the purpose of forming aldehyde and carboxylic groups, consequently representing binding sites for protonated amino groups of chitosan in the form of a solution, or in the form of nanoparticles presented afterwards onto the fiber surfaces. Among different oxidation procedures periodate oxidation, which resulting in opening of the pyranose ring and introduction of aldehydes at both C-2 and C-3 positions, was chosen mainly due to the significant advantages of periodates over other oxidizing agents, as they minimize degradation and retain the mechanical and morphological properties of the cellulose fibers. Furthermore, the resulting compound 2,3-dialdehyde cellulose can be used to immobilize proteins $[14,15]$ or amino polysaccharides $[16,17]$ by reaction with their amino functions, or as ion-exchange materials after further oxidation of the aldehydes to the corresponding carboxylic acids [18]. Although several publications exists on this topic [14-18], there were no detailed studies regarding desorption of chitosan from cellulose or activated cellulose and the latest represent the innovative part of the research. In addition, to our knowledge, there was no comparison done between adsorption of chitosan nanoparticles via chitosan macromolecules onto cellulose viscose fibers. Even more, antimicrobial properties of viscose fibers functionalized by chitosan nanoparticles were not presented yet.

Thus, most important was to study the influence of formed oxidized groups on the stability of chitosan binding onto cellulose. Desorption of chitosan/chitosan nanoparticles from the fiber surfaces was evaluated by several techniques. In order to achieve permanent antimicrobial properties, as well as a satisfactory amount of anchoring sites in/on the fibers onto which chitosan could permanently bind at the same time, a satisfactory amount of free amino groups of chitosan, which are responsible for antimicrobial activity, is needed.

In this context, antimicrobial action against different pathogens' microorganisms, namely, Staphylococcus Aureus, Escherichia Coli and Candida Albicans was

Journal of Engineered Fibers and Fabrics

Volume 10, Issue 2 - 2015 evaluated after samples being functionalized with chitosan/chitosan nanoparticles.

\section{EXPERIMENTAL}

Materials and Methods Fibers Specification

Investigations were carried out on $100 \%$ viscose fibers (Lenzing AG, Austria) of titer 2.8 dtex and length $30 \mathrm{~mm}$.

\section{Viscose Functionalization}

Oxidation:

Cellulose fibers were immersed in a solution of sodium periodate containing $0.1 \mathrm{M}$ acetic acid buffer (ratio of $1: 50, \mathrm{v} / \mathrm{v}$ ) at $\mathrm{pH}$ of 4.0 , and a concentration of $4.0 \mathrm{mg} / \mathrm{mL}(0.4 \%(\mathrm{w} / \mathrm{v}))$.

The fibers were then stirred gently in the absence of light, under room conditions for a designed time. Short oxidation time (20 min.) was selected on the base of our previous research $[16,19]$ with the aim to achieve the sufficient aldehyde group content mainly in the easy-to-access portion of viscose fibers. Short oxidation time is needed in order to prevent impact of periodate on the crystalline regions of cellulose, which means that only in this case huge molecules of chitosan /chitosan nanoparticles are able to access aldehyde groups of viscose. After oxidation was completed the cellulose samples were transferred onto filter paper and thoroughly rinsed with the use of icy cold distilled water through a Buchner funnel for the purpose of removing any oxidant. The oxidized cellulose samples were then dried for $72 \mathrm{~h}$ at a room temperature. The characteristics of the fibers are given in Table I.

Chitosan solution preparation:

Chitosan w/w $=0.5 \%$ (Kitozyme, Belgium) of molecular weight: 8.2000 and degree of acetylation: $22.4 \%$ was used. The calculated amount of chitosan in the form of powder was suspended into Milli-Q water (ultra-pure water), with added concentrated lactic acid (LaAC) to set a pH of 3.6. 24 hour of stirring at room temperature was needed to dissolve the chitosan completely i.e. to obtain a solution of chitosan.

Nano-particles of chitosan:

Sodium tripolyphosphate (TPP) was used to prepare the chitosan nanoparticles. The nanoparticles were prepared in accordance with the known procedure of so-called ionic gelation [20- 23]. Polyelectrolyte polymers such as chitosan have the ability to form gel when polyvalent, and oppositely-charged ions like TPP are added into the solution. With this ionic interaction cross-linking occurs forming a 
polyelectrolyte complex in the form of nanoparticles [24]. Previously prepared solution of TPP of known concentration $(1 \mathrm{mg} / \mathrm{mL})$ was added to the chitosan solution $(\mathrm{w} / \mathrm{w}=0.5 \%)$ in the volume ratio of $1: 1$. This formation was mixed afterwards for one hour by using a magnetic stirrer until chitosan nanoparticles formed spontaneously within the range of 200-300 $\mathrm{nm}$, which was proved by scanning electron microscopy (SEM analysis) in previous work [24].

Impregnation of cellulose fibers with chitosan and chitosan nanoparticles:

A non-oxidized (A) and an oxidized sample (B) with characteristics collected in Table I were used for the cellulose fibers' functionalization.

TABLE I. Oxidized cellulose characteristics.

\begin{tabular}{|c|c|c|c|c|}
\hline Sample & \multicolumn{2}{|c|}{ A } & \multicolumn{2}{|c|}{ B } \\
\hline Reaction time [min] & & & 2 & \\
\hline $\begin{array}{l}\text { Water retention [\%] and } \\
\mathrm{cV}^{*}[\%]\end{array}$ & 52.31 & 2.69 & 45.30 & 3.69 \\
\hline $\begin{array}{l}\text { Iodine sorption (ISV) [mg } \\
\left.\mathrm{I}_{2} / \mathrm{g}\right] \text { and } \mathrm{cV}^{*}[\%]\end{array}$ & 168.70 & 0.24 & 140.90 & 0.00 \\
\hline $\begin{array}{l}\text {-COOH groups }[\mu \mathrm{mol} / \mathrm{g}] \\
\text { and } \mathrm{cV}^{*}[\%]\end{array}$ & 46.63 & 1.08 & 47.03 & 3.02 \\
\hline $\begin{array}{l}\text {-CHO groups }[\mu \mathrm{mol} / \mathrm{g}] \\
\text { and } \mathrm{cV}^{*}[\%]\end{array}$ & 14.84 & 19.16 & 193.60 & 4.80 \\
\hline
\end{tabular}

\section{Viscose Functionalization with Chitosan}

The sample of oxidized cellulose fibers $(\mathrm{m}=2 \mathrm{~g})$ was immersed into a prepared solution of chitosan or chitosan nanoparticles, respectively $(\mathrm{V}=100 \mathrm{~mL}, \mathrm{pH}$ = 5) for $30 \mathrm{~min}$. Then, the sample of fibers was filtered through a funnel, and dried by using a laboratory oven at a temperature of $70{ }^{\circ} \mathrm{C}$, and time of 30 minutes. An identical procedure was also used for cellulose non-oxidized fibers' functionalization. Table II contains abbreviations of the samples.

TABLE II. Abbreviation and sample description.

\begin{tabular}{ll}
\hline Abbreviation & Sample description \\
\hline A & Reference/Non-oxidized cellulose \\
B & Oxidized cellulose \\
C & B + chitosan \\
D & B + chitosan nanoparticles \\
E & A + chitosan \\
F & A + chitosan nanoparticles
\end{tabular}

Journal of Engineered Fibers and Fabrics

Volume 10, Issue 2 - 2015

\section{Analytical Methods}

$X$-ray photoelectron spectroscopy XPS, (Atomic and elemental composition of fiber surface)

The spectra were recorded using the PHI model TFA XPS spectrometer. The atomic composition was measured after the chitosan treatment, and then compared to the elemental chemical composition of the non-treated material's surface. The base pressure in the XPS analysis chamber was about $6 \times 10^{-10} \mathrm{mbar}$ and the samples were excited with $\mathrm{X}$-rays over a specific $400 \mu \mathrm{m}$ area using monochromatic $\mathrm{Al} \mathrm{K}_{\alpha 1,2}$ radiations at $1486.6 \mathrm{eV}$. The photoelectrons were detected by a hemispherical analyzer, positioned at an angle of $45^{\circ}$ with respect to the sample's surface. Energy resolution was about $0.6 \mathrm{eV}$. Spectra were recorded from three locations on each sample using an analysis area of $400 \mu \mathrm{m}$. Two repetitions for each sample were done. The surface elemental concentrations were calculated from the survey -scan spectra using the Multipak program.

\section{Gravimetric Measurements of Sample Mass}

The exact amount of fiber samples were dried in a weighing glass container at $120{ }^{\circ} \mathrm{C}$ for 4 hours, and afterwards cooled in a desiccator. All the samples were weighed for the purpose of comparing their weights with those of the untreated reference fibers. The final weight differences between the non-treated and treated samples showed a successful binding of the formulation onto the viscose fibers.

\section{Desorption Procedure}

\section{Total Nitrogen Content (TN)}

$1 \mathrm{~g}$ of the fibers was treated for 4 hours in $50 \mathrm{~mL}$ of aqueous solution $(\mathrm{pH}=3.6$, adjusted by $0.1 \mathrm{M}$ hydrochloric acid). The solution was filtered and the filtrate was subjected to TN measurements using the procedure described by Goyal et al. [25] in a Multi N/C 2100 S (Analytik Jena AG) instrument. The results were expressed as mmol of nitrogen per kilogram of sample.

\section{Polyelectrolyte Titration (Desorbed Chitosan)}

The analyte was composed from a filtrated solution (desorption bath) of $40 \mathrm{~mL}$ with addition of few drops of indicator Toluidine blue O (Sigma Aldrich) with the $\mathrm{pH} \approx 3.6$ (adjusted by $0.1 \mathrm{M}$ hydrochloric acid). A Mettler Toledo DL 53 titrator, with a $10 \mathrm{~mL}$ burette was used for the incremental addition of the polyelectrolyte titrant (PES-Na; c $=10 \mathrm{mM}$ ). The incremental additions of $25 \mu \mathrm{L}$ were added every 3 10 seconds. The absorbance was measured as a potential change in $\mathrm{mV}$, using a Mettler Toledo Phototrode DP660 at a wavelength of $660 \mathrm{~nm}$. The concentration of the protonated amino groups as a consequence of desorbed chitosan was determined 
from the equivalent volume of the added PES-Na solution, detected as the steep step in the absorbance vs $V(\mathrm{PES}-\mathrm{Na}$ ) titration curve, and by estimating a $1: 1$ binding stoichiometry of ethylenesulfonate to chitosan amino groups. Two replicates of polyelectrolyte titrations were performed for each sample. The example of polyelectrolyte titration curve is given in Figure 1.

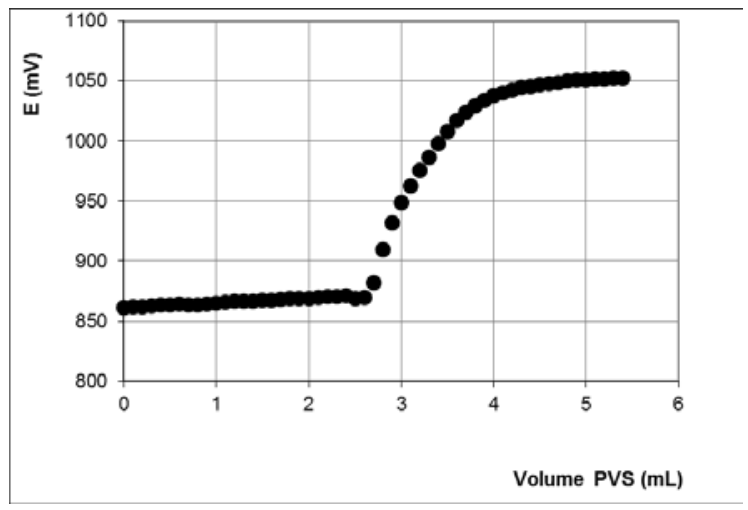

FIGURE 1. The example of polyelectrolyte titration curve of chitosan solution. The equivalent point $(\mathrm{mmol} / \mathrm{kg})$ is determined from the slope of the curve.

\section{Antimicrobial Activity}

The antimicrobial properties of the functionalized viscose fibers were evaluated by a modified method of ASTM E2149-01, which is a quantitative antimicrobial test method performed under dynamic contact conditions. Gram-positive and Gram-negative bacteria (Table VII), as well as fungi, were used as test organisms. The selected micro-organism presented a standard base of pathogen microorganism. An incubated test culture in a nutrient broth was diluted using a sterilized $0.3 \mathrm{mM}$ phosphate buffer $\left(\mathrm{KH}_{2} \mathrm{PO}_{4} ; \mathrm{pH}=6.8\right)$ in order to provide a final concentration of $1.5-3.0 \cdot 10^{5}$ colony forming units (CFU)/mL. This solution was used as a working bacterial dilution. Each sample $(0.5-2.0 \mathrm{~g})$ was cut into small pieces $(1 \mathrm{x} 1 \mathrm{~cm})$ and transferred to a $250 \mathrm{~mL}$ Erlenmeyer flask containing $50 \mathrm{~mL}$ of the working bacterial dilution. All the flasks were loosely capped, placed in the incubator, and shaken for $1 \mathrm{~h}$ at $37^{\circ} \mathrm{C}$ and $120 \mathrm{rpm}$ using a Wrist Action incubator shaker. After a series of dilutions using the buffer solutions, $1 \mathrm{~mL}$ of the diluted solution was plated in nutrient agar. The inoculated plates were incubated at $37^{\circ} \mathrm{C}$ for $24 \mathrm{~h}$ and the surviving cells counted. The average values of the duplicates were, after multiplying by the dilution factor, converted into CFU/mL in the flasks. The antimicrobial activity was expressed as a $\mathrm{R}=\%$ reduction of the organism after contact with the test specimen, compared to the number of bacterial cells surviving after contact with the control [26, 28].

\section{RESULTS \\ Adsorption \\ XPS}

From the Table III it is possible to conclude about the chemical composition of oxidized cellulose fibers' surface as well as those functionalized by chitosan. First, it can be seen that the percent of oxygen is maximal in the case of oxidized fibers which reflects the emergence of new aldehyde groups as clearly seen from the Table II. An increase of oxygen onto the surface of the sample B is not as significant. This is attributable to the fact that most of the oxidized groups are formed in the internal part of the fibers and not onto their surfaces. It is known that during oxidation relatively small ions of periodate penetrate into the interior of the cellulose, and oxidizes the glucose units of C2 and C3, so that most of the newly formed- CHO groups are located inside the fibers [7]. This may be proved by the fact that the number of aldehyde groups increases from $14.84 \mu \mathrm{mol} / \mathrm{g}$ to $193.60 \mu \mathrm{mol} / \mathrm{g}$ determined by potentiometric titration, as already discussed [14] and as it listed in Table I.

It is evident from the Table III that the percent of carbon of all cellulose-chitosan functionalized samples increased, while the oxygen content of chitosan/chitosan nanoparticles treated samples is reduced minimally when compared to the reference (non-oxidized cellulose). The increase in percent of carbon indicates the successful adsorption of chitosan macromolecules onto the surface of cellulose fibers. The most important, results of atomic nitrogen concentrations indicate that chitosan is successfully bounded onto the surface of the fibers.

It can be seen from the results that the concentration of nitrogen in percentage is approximately $2 \mathrm{x}$ higher in the example of a sample $C$ (oxidized cellulose + chitosan) if compared with the samples D (oxidized cellulose + chitosan nanoparticles), and E (nonoxidized cellulose + chitosan), respectively. Obviously, in the case of non-oxidized and oxidized cellulose strong electrostatic interactions are involved in binding mechanisms of chitosan due to anionic character of cellulose (carboxyl group content of both samples is about $47 \mu \mathrm{mol} / \mathrm{g}$ ) and positively charged amino groups of chitosan (impregnation of cellulose fibers with chitosan was done at $\mathrm{pH}$ lower than its pKa 6.3). 
Furthermore, in the case of oxidized cellulose chemical bounds between the amino and aldehyde groups could be established which is manifested into the most intensive adsorption (electrostatic attraction) of chitosan onto those oxidized fibers as already seen in [13]. Previously oxidized sample (C) express after chitosan binding almost double amount of nitrogen if compared to non-oxidized.

In the case of chitosan nanoparticles which are of size about $200-300 \mathrm{~nm}$, as pointed out in previous research in [24], besides binding onto the surface of oxidized cellulose, penetration of nanoparticles into the interior of the fibers being expected as well. The latter cannot be detected with using the XPS technique since this analysis is limited to a relatively thin surface layer (approx. $10 \mathrm{~nm}$ ). However, in the case of chitosan nanoparticles binding, the adsorption onto the surface is higher with non-oxidized cellulose fibers (sample F) in comparison with the oxidized one (sample D).

TABLE III. Chemical compositions of fibers' surfaces (atomic \% of $\mathrm{C}, \mathrm{N}, \mathrm{O}$, and $\mathrm{Si}$ ).

\begin{tabular}{lllll}
\hline Abbr. & \% C & \% N & \% O & \% Si \\
\hline A & $60.4 \pm 0.3$ & - & $32.5 \pm 1.1$ & - \\
B & $64.8 \pm 1.0$ & $0.9 \pm 0.1$ & $34.5 \pm 1.4$ & $1.0 \pm 0.5$ \\
C & $68.1 \pm 0.3$ & $3.4 \pm 0.1$ & $28.5 \pm 0.5$ & - \\
D & $66.4 \pm 0.4$ & $1.9 \pm 0.4$ & $30.2 \pm 0.7$ & $1.4 \pm 0.4$ \\
E & $67.1 \pm 0.5$ & $1.6 \pm 0.1$ & $30.0 \pm 0.5$ & $1.4 \pm 0.3$ \\
F & $64.4 \pm 0.6$ & $2.6 \pm 0.1$ & $33.0 \pm 1.2$ & -
\end{tabular}

\section{Gravimetric Method}

Table IV shows masses of cellulose fibers before and after the adsorption of chitosan and chitosan nanoparticles, respectively.

The mass of fibers is higher after, as prior treatment, due to chitosan/chitosan nanoparticles binding onto the surface, after the fibers have been functionalized. This is proof of successful binding.

TABLE IV. The mass of the samples before and after functionalization by chitosan or chitosan nanoparticles.

\begin{tabular}{llll}
\hline Abbr. & $\begin{array}{l}\text { Mass before } \\
\text { function. }[\mathbf{g}]\end{array}$ & $\begin{array}{l}\text { Mass after } \\
\text { function. } \\
\text { [g] }\end{array}$ & $\begin{array}{l}\text { Difference } \\
\text { in masses- } \Delta \mathbf{m} \text { [g] }\end{array}$ \\
\hline C & 2.1738 & 2.3041 & 0.1303 \\
D & 2.1667 & 2.2777 & 0.1110 \\
E & 2.0033 & 2.1516 & 0.1483 \\
F & 2.0007 & 2.0818 & 0.0811
\end{tabular}

It can also be seen that the difference in masses is the highest for the case when chitosan from the solution was adsorbed onto the cellulose surface, when compared to the chitosan nanoparticles. Approximately the same quantity of chitosan from the solution is adsorbed onto oxidized, as well as on the non-oxidized cellulose fibers (samples C, and D), while in the case of chitosan nanoparticles, the adsorption is slightly higher for the oxidized cellulose fibers (sample E) than for non-oxidized.

When this method is compared to XPS the trend of results differs. This is due to different nature of techniques. XPS detect only limited surface amount of nitrogen as a consequence of chitosan binding, whilst the differences in masses are connected to the total amount of bounded chitosan in the inner as well as in the external fiber part. With the differences in masses one can detect chitosan macromolecules attached onto the fiber surface, surface bounded nanoparticles and those that may penetrate into fiber pores. The latest, as already mentioned, is out of the XPS detection.

\section{Desorption}

\section{Total nitrogen (TN)}

Table $V$ shows the presence of total nitrogen (TN) within the desorption baths $(8 \mathrm{~h}$, slightly acidic $\mathrm{pH}$, $\mathrm{pH}=5.9)$. Surprisingly nitrogen was detected in the desorption baths of reference as well as oxidized cellulose fibers. Obviously in both fibers, impurities in the form of a releasable low molecular fraction of nitrogen origin are presented. For the oxidized cellulose fibers which have been further functionalized with chitosan (C)/chitosan nanoparticles (D), a less amount of desorbed chitosan is detected within the bath, as in the case of nonoxidized cellulose fibers which have been further functionalized with chitosan (E)/chitosan nanoparticles (F). When comparing samples $\mathrm{C}$ and $\mathrm{E}$ (chitosan from solution was attached on the fiber surface), it can be seen that in the example of nonoxidized sample $2.5 \mathrm{x}$ more of chitosan is released into the water bath. This trend is even more intense in the case of chitosan nano-particles.

TABLE V. Total nitrogen (TN).

\begin{tabular}{llll}
\hline Abbr. & TN $[\mathbf{m g} / \mathbf{L}]$ & $\mathbf{m m o l} / \mathbf{L}$ & $\mathbf{~ m m o l} / \mathbf{g}$ \\
\hline $\mathrm{A}$ & 6.90 & 0.49 & 0.049 \\
$\mathrm{~B}$ & 5.90 & 0.42 & 0.042 \\
$\mathrm{C}$ & 15.20 & 1.09 & 0.109 \\
$\mathrm{D}$ & 6.99 & 0.49 & 0.049 \\
$\mathrm{E}$ & 37.52 & 2.68 & 0.268 \\
$\mathrm{~F}$ & 23.32 & 1.66 & 0.166
\end{tabular}

With the non-functionalized cellulose, the chitosan nano-particles are $4 \mathrm{x}$ more intensively released if compared to the oxidized cellulose (as a carrier). The results clearly show situation that desorption is much more pronounced in the case of non-oxidized 
cellulose fibers, especially ones which have been further functionalized with chitosan solution (E).

\section{Gravimetric Method}

Table VI shows the mass of fibers before and after desorption. The results show a lower mass of the samples after desorption due to antimicrobial active compound release during desorption of the fibers within an aqueous medium. The greater amount of polymer (chitosan or, chitosan nano-particles) was desorbed in the case of non-oxidized fibers; the results being in the line with the results of polyelectrolyte titration, and $\mathrm{TN}$, respectively.

TABLE VI. The mass of the samples before and after desorption.

\begin{tabular}{llll}
\hline Abbr. & $\begin{array}{l}\text { Mass before } \\
\text { desorption [g] }\end{array}$ & $\begin{array}{l}\text { Mass after } \\
\text { desorption } \\
\text { [g] }\end{array}$ & $\begin{array}{l}\text { Difference in } \\
\text { masses- } \Delta \mathbf{m} \\
\text { [g] }\end{array}$ \\
\hline $\mathrm{C}$ & 0.2504 & 0.2392 & 0.0112 \\
$\mathrm{D}$ & 0.2509 & 0.2486 & 0.0023 \\
$\mathrm{E}$ & 0.2510 & 0.2346 & 0.0164 \\
$\mathrm{~F}$ & 0.2499 & 0.2458 & 0.0041
\end{tabular}

For the reference sample (A) no differences in mass were obtained after desorption experiment. All of three methods used for analyzing of desorption clearly show higher stability of chitosan binding in the case of oxidized cellulose.

Obviously electrostatic attraction and physical interactions (such as hydrogen bonds, hydrophobic interaction) between non-oxidized cellulose (as an adsorbent) and chitosan (as an adsorbate) are not enough to establish permanent binding. This research clearly suggest that chemical bounds $(-\mathrm{N}=\mathrm{CH})$, which could be formed between aldehyde groups of oxidized cellulose and (deprotonated) amino groups of chitosan as proved in similar previous research [29], enables more stable coating of chitosan onto fiber surface. It has to be noted that at $\mathrm{pH}=5$ (adsorption conditions) around $70 \%$ of amino groups are protonated (pK value of chitosan is around 6.3) [28] and those groups are available for electrostatic interactions with partially deprotonated carboxyl groups. Residual $30 \%$ of deprotonated amino groups are ideal for Schiff base formation (with fiber carboxyl groups). Due to the fact that both, nonoxidized and oxidized cellulose fibers possess the same amount of carboxyl groups (Table II), it can be concluded that aldehyde groups introduced after oxidation, which may be fully occupied (chemically bounded) by those $30 \%$ of deprotonated amino groups, present an advantage regardless the stability of chitosan coating.

Journal of Engineered Fibers and Fabrics

Volume 10, Issue 2 - 2015

\section{Antimicrobial Testing}

In accordance with the used standard the sample shows anti-microbial action if the reduction is greater than $75 \%$. Table VII shows the results of antimicrobial testing, where it can be seen that the antimicrobial effect of almost all the samples of fibers increases in comparison with the reference. It can also be seen that oxidized fibers as a matrix for further functionalization reduce antimicrobial action. So, if we compare sample C (oxidized fibers + chitosan) with sample E (non-oxidized fibers + chitosan), a better reduction of selected microorganisms is seen with the sample E. Both reduce the growth of bacteria Staphylococcus Aureus and fungi Candida Albicans. Sample E reduces (R (\%)) microorganism Eschericha Coli better as the sample C. The same trend can be seen by comparing samples D (oxidized cellulose + chitosan nano-particles), and F (non-oxidized cellulose + chitosan nano-particles). However, the results of oxidized, functionalized fibers are surprising. Considering the nature of the binding a reduction in the available protonated amino groups would be expected resulting in a loss of antimicrobial action. Apparently, in spite of chemical binding, a sufficient quantity of these groups remains for sufficient antimicrobial effect on the specific micro-organisms.

TABLE VII. Antimicrobial testing.

\begin{tabular}{llll}
\hline Abbr. & $\begin{array}{l}\text { Staphylococcus } \\
\text { Aureus }\end{array}$ & $\begin{array}{l}\text { Escherichia } \\
\text { Colli }\end{array}$ & $\begin{array}{l}\text { Candida } \\
\text { Albicans }\end{array}$ \\
\hline A & $64 \%$ & $22 \%$ & $1 \%$ \\
B & $68 \%$ & $43 \%$ & $12 \%$ \\
C & $88 \%$ & $33 \%$ & $97 \%$ \\
D & $82 \%$ & $71 \%$ & $85 \%$ \\
E & $94 \%$ & $71 \%$ & $98 \%$ \\
F & $98 \%$ & $91 \%$ & $86 \%$
\end{tabular}

\section{CONCLUSION}

With the use of XPS spectroscopy, TN, polyelectrolyte titrations, and gravimetric method, by which the results of functionalization by chitosan, and chitosan nano-particles were evaluated, it is confirmed the hypothesis that desorption of chitosan as well as chitosan nano-particles is negligible in the cases of oxidized samples when compared to nonoxidized ones.

Oxidized cellulose possesses functional groups which are suitable for the chemical binding of chitosan and chitosan nano-particles onto it and thus result into the fact that higher amount of chitosan is irreversibly bonded as in the case of non-oxidized sample. 
With the help of anti-microbial analysis, it was discovered that previous oxidation does not deteriorates the antimicrobial efficiencies of the functionalized fibers even due to the possible blocking of amino groups because of possible chemical binding.

The procedure used may have reveled several applications for antimicrobial textiles that prefer permanent active coating such as sport textiles, underwear, and socks.

\section{REFERENCES}

[1] Subhash, A. et al., Medical textiles and biomaterials for healthcare, Woodhead Publishing, (2005).

[2] Fras, L. et al., Analysis of the oxidation of cellulose fibres by titration and XPS, Colloids surf., A Physicochem. eng. Asp,, 260 (1-3), 101-108 (2005).

[3] Windler, L., et al., Comparative evaluation of antimicrobials for textile applications, Environment International, 53, 62-73 (2013).

[4] Shahid-ul-Islam, et al., Green Chemistry Approaches to Develop Antimicrobial Textiles Based on Sustainable BiopolymersA Review, Ind. Eng. Chem. Res., 52 (15), 5245-5260 (2013).

[5] Gao, Y. and Cranston, R., Recent Advances in Antimicrobial Treatments of Textiles, Textile Research Journal, 78 (1), 60-72 (2008).

[6] Mahbubul, B., M. and Mubarak, K., A., An Overview on Surface Modification of Cotton Fiber for Apparel Use, Journal of Polymers and the Environment, 21 (1), 181-190 (2013).

[7] Munda, M., Elementary characterization of functionalized fibres, Diploma work, Maribor (2011).

[8] Kerec-Kos, M., Use of chitosan in pharmacy, Farmacevtski vestnik, 57 (5), 287-291 (2006).

[9] Singla, A. K. and Chawla, M., Chitosan: some pharmaceutical and biological aspects - an update, Journal of Pharmacy and Pharmacology, 53 (8), 1047-1067 (2001).

[10] Strnad, S. et al., Chitosan - a universally applicable biopolymer, Tekstilec, 50 (10/12), 243-261 (2007).

[11] Rabea, E. I., Badawy, M. E. T., Stevens, C. V., et al., Chitosan as Antimicrobial Agent, Applications and Mode of Action, Biomacromolecules, 4 (6), 1457-1465 (2003).

[12] Ravi Kumar, M. N. V., A review of chitin and chitosan applications, Reactive and Functional Polymers, 46 (1), 1-27 (2000).
[13] Strnad, S. et al., Cellulose fibres functionalised by chitosan : characterization and application, V: ELNASHAR, Magdy M. (ur.), Biopolymers. Rijeka: Sciyo, cop., 181-200 (2010).

[14] Nikolic, T. et al., Sodium periodate oxidized cotton yarn as carrier for immobilization of trypsin, Carbohydrate Polymers, 82 (3), 976981 (2010).

[15] Varavinit, S. et al., Covalent immobilization of a glucoamylase to bagasse dialdehyde cellulose, World Journal of Microbiology \& Biotechnology, 17, 721-725 (2001).

[16] Janjic, S. et al., Biologically active fibers based on chitosan-coated lyocell fibers, Carbohydrate Polymers, 78 (2), 240-246 (2009).

[17] Strnad, S. et al., Influence of Chemical Modification on Sorption and Mechanical Properties of Cotton Fibers Treated with Chitosan, Textile Research Journal, 78 (5), 390-398 (2008).

[18] Kim, U.J. and Kuga, S., Ion-exchange separation of proteins by polyallyllaminegrafted cellulose gel, Journal of Chromatography A, 955, 191-196 (2002).

[19] Potthast, A. et al., Studies on oxidative modifications of cellulose in the periodate system: Molecular weight distribution and carbonyl group profiles, Holzforschung, 61 (6), 662-667 (2007).

[20] Bodnar, M. et al., Preparation and characterization of chitosan-based nanoparticle, Biomacromolecules, 6(5), 25212527 (2005).

[21] Gan, Q. and Wang, T., Chitosan nanoparticle as protein delivery carrier-Systematic examination of fabrication conditions for efficient loading and releas, Colloids and Surfaces B: Biointerfaces, 59 (1), 24-34 (2007).

[22] Kunjachan, S. and Jose, S., Understanding the mechanism of ionic gelation for synthesis of chitosan nanoparticles using qualitative techniques, Asian J Pharm [serial online], 4 (2), 148-153 (2010).

[23] Gan, Q. et al.., Modulation of surface charge, particle size and morphological properties of chitosan-TPP nanoparticles intended for gene delivery, Colloids Surf B Biointerfaces, 44 (23), 65-73 (2005).

[24] Qi, L., Xu, Z., Jiang, X., et al., Preparation and antibacterial activity of chitosan nanoparticles. Carbohydrate Research,, 339(16):,2693-2700 (2004). 
[25] Goyal, S. et al., Chemical and biological changes during composting of different organic wastes and assessment of compost maturity, Bioresource Technol., 96, 14, 158491 (2005)

[26] ASTM E2149-10 Standard Test Method for Determining the Antimicrobial Activity of Immobilized Antimicrobial Agents Under Dynamic Contact Conditions.

[27] Sakai, Y. et al., Chitosan-coating of Cellulosic materials using an aqueous chitosan- $\mathrm{CO}_{2}$ solution, Polym J., 34, (3), 144-148 (2002).

[28] Fras, L., Ristić, T., Tkavc, T., Adsorption and Antibacterial Activity of Soluble and Precipitated Chitosan on Cellulose Viscose Fibers, Journal of Engineered Fibers and Fabrics, 7 (1), 50-57 (2012).

[29] Fras, L., Kokol, V., Čakara, D., Antimicrobial and antioxidant properties of chitosan-based viscose fibres enzymatically functionalized with flavonoids. Tex. res. j., 81(15), 15321540 (2011).

\section{AUTHORS' ADDRESSES \\ Olivera Sauperl \\ Mirjana Kostic \\ Jovana Milanovic \\ Lidija Fras Zemljic}

Faculty of Mechanical Engineering

Smetanova ulica 017

Maribor, Slovenia SI 2000

SLOVENIA 